\title{
Beyond the model of conductor with fixed cross-section and length: exactly and numerically solvable problems
}

\author{
Vladimir Ivchenko ${ }^{* 1]}$ \\ ${ }^{1}$ Kherson State Maritime Academy, Department of Natural Sciences Training, Kherson 73000, Ukraine
}

Received on 09 May 2020. Accepted on 01 October 2020.

\begin{abstract}
In this paper we present an overview of methods of resistance finding for the conductor with variable crosssection or (and) length. First, we consider the cases of the finite size resistors and an infinite homogeneous weakly conducting medium with two electrodes. Next, we continue the Romano and Price analysis about the truncated cone problem by means of comprehensive numerical calculations done for the trapezoid plates. We conclude that in general case of a conductor with variable cross-section the homogeneous electric field approximation gives only the lower limit estimation for the resistance value. This fact can be explained on the basis of the minimum electric power principle. The issues outlined in this article will be useful for advanced undergraduates, who study methods for solving electrostatic problems.
\end{abstract}

Keywords: problem solving, electrical resistance, trapezoid plate problem.

\section{Introduction}

It is well known that the electrical resistance $R$ of an object is defined as the proportionality constant between voltage $U$ and current $I$ :

$$
R=\frac{U}{I}
$$

The electrical resistance of a uniform conductor is given by relation [1]:

$$
R=\rho \frac{l}{A},
$$

where $\rho$ is the electrical resistivity; $l$ is the length of the conductor; $A$ is the cross-sectional area of the conductor. It should be noted that Equation (2) is applicable only for a conductor with a constant cross-section (wherein this cross-section may have any shape) along its length. In this paper we consider the examples allowing analytical or only numerical solution of the problem of resistance finding for the conductor with variable cross-section (length) or conductor without clear boundaries. The issues outlined in this article will be useful for advanced undergraduates, who study methods for solving electrostatic problems.

\section{Analytically Solvable Problems}

Let us consider a hollow conducting ball with inner and outer radii equal to $r_{1}$ and $r_{2}$ respectively. It is necessary to find its resistance, if voltage is applied between

\footnotetext{
* Correspondence email address: reterty@gmail.com
}

the inner and outer surfaces. We note that in this case the current flow lines are radial straight lines (see Fig. 1). Using this fact one can split the conductor into infinitesimal spherical layers of thickness $\mathrm{d} r$ and radius $r$. According to Equation (2) each of them has infinitesimal resistance $\mathrm{d} R=\rho \mathrm{d} r /\left(4 \pi r^{2}\right)$. Since this layers are connected in series, we have:

$$
R=\int \mathrm{d} R=\frac{\rho}{4 \pi} \int_{r_{1}}^{r_{2}} \frac{\mathrm{d} r}{r^{2}}=\frac{\rho}{4 \pi}\left(\frac{1}{r_{1}}-\frac{1}{r_{2}}\right) .
$$

If $r_{2} \rightarrow \infty$ (charged ball with radius $r_{1}$ placed in an infinite conducting medium with resistivity $\rho$ ), then from Equation (3) we get: $R=\rho /\left(4 \pi r_{1}\right)$.

Now we consider a long hollow cylindrical conductor of length $h$ and with inner and outer radii equal to $r_{1}$ and $r_{2}\left(r_{2} \ll h\right)$ respectively. As in the previous case its

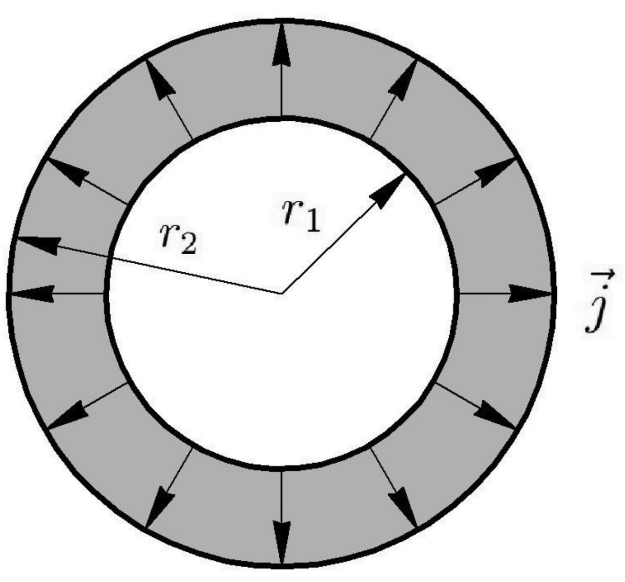

Figure 1: A hollow conducting ball. 


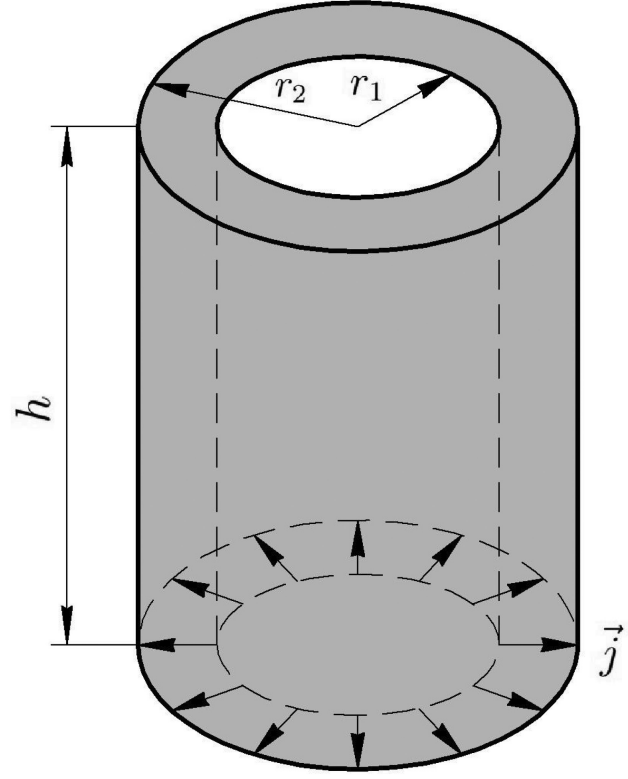

Figure 2: A hollow cylindrical conductor.

current flow lines are radial straight lines (see Fig. 2). Then splitting this conductor into infinitesimal cylindrical shells with thickness $\mathrm{d} r$, we have: $\mathrm{d} R=\rho \mathrm{d} r /(2 \pi h r)$ and

$$
R=\int \mathrm{d} R=\frac{\rho}{2 \pi h} \int_{r_{1}}^{r_{2}} \frac{\mathrm{d} r}{r}=\frac{\rho}{2 \pi h} \ln \left(\frac{r_{2}}{r_{1}}\right)
$$

If $r_{2} \rightarrow \infty$ (long charged cylinder of radius $r_{1}$ placed in an infinite conducting medium with resistivity $\rho$ ), then from Equation (4) we get: $R \rightarrow \infty$.

The above-considered method for determining the resistance by summing its value over infinitesimal layers, is also valid for the case, where the current flow lines are bent (due to conductor bending), but remain parallel in any cross-section of a conductor. As an example we may consider the half ring with thickness $t$ shown in Fig. 3. Let us try to find the resistance between its ends. In this case we deal with the conductor of variable length. The resistance of an infinitesimal annulus with radius $r$ and width $\mathrm{d} r$ is $\mathrm{d} R=\rho \pi r /(t \mathrm{~d} r)$. The resistances from different sub-annuli are in parallel with one another. Therefore,

$$
\frac{1}{R}=\int \frac{1}{\mathrm{~d} R}=\frac{t}{\pi \rho} \int_{r_{1}}^{r_{2}} \frac{\mathrm{d} r}{r}=\frac{t}{\pi \rho} \ln \left(\frac{r_{2}}{r_{1}}\right)
$$

\section{The Resistor with no Clear Boundaries}

The above-described method for determining the resistance of an object fails, when the conductor does not have clear boundaries ("fuzzy conductor" or "massive conductor"). Let us consider a system of two conductors of arbitrary shape, which are placed in an infinite homogeneous weakly conducting medium with resistivity $\rho$ and permittivity $\epsilon$ [2. Now we find product $R C$, where $R$ is the medium resistance between conductors and $C$ is the mutual capacitance of this system. Since the medium is weakly conductive, we can assume that the conductivity of conductors is much higher than the conductivity of the medium. Then we can neglect the change in potential within the conductors, that is, we assume that all the points of each electrode have the same potential. We keep the voltage between electrodes constant and equal to $U$.

We mentally surround one of the electrodes with an arbitrary closed surface $S$. Then the total current through this surface is:

$$
I=\oiint_{S} \vec{j} \cdot \mathrm{d} \vec{A}=\frac{1}{\rho} \oiint_{S} \vec{E} \cdot \mathrm{d} \vec{A}
$$

where $\vec{j}$ is the current density; $\vec{E}$ is the electric field strength (here we used relation $\vec{j}=\vec{E} / \rho$ ). On the other hand, applying the Gauss's law, we obtain:

$$
\oiint_{S} \vec{E} \cdot \mathrm{d} \vec{A}=\frac{q}{\epsilon \epsilon_{0}}
$$

where $q=C U$ is the charge of one of the electrodes; $\epsilon_{0}$ is the vacuum permittivity. Using Eqs. (1), (6), (7), we finally derive 2]:

$$
R C=\rho \epsilon \epsilon_{0}
$$

As an example of using Equation (8) we consider the case of two parallel wires each of radius $r$ and length $l$. Let the distance between their axes of symmetry be

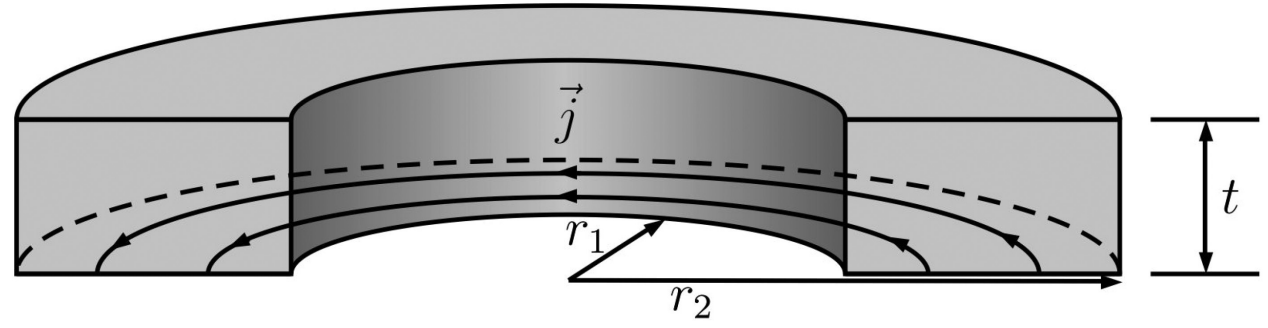

Figure 3: A conducting half ring 


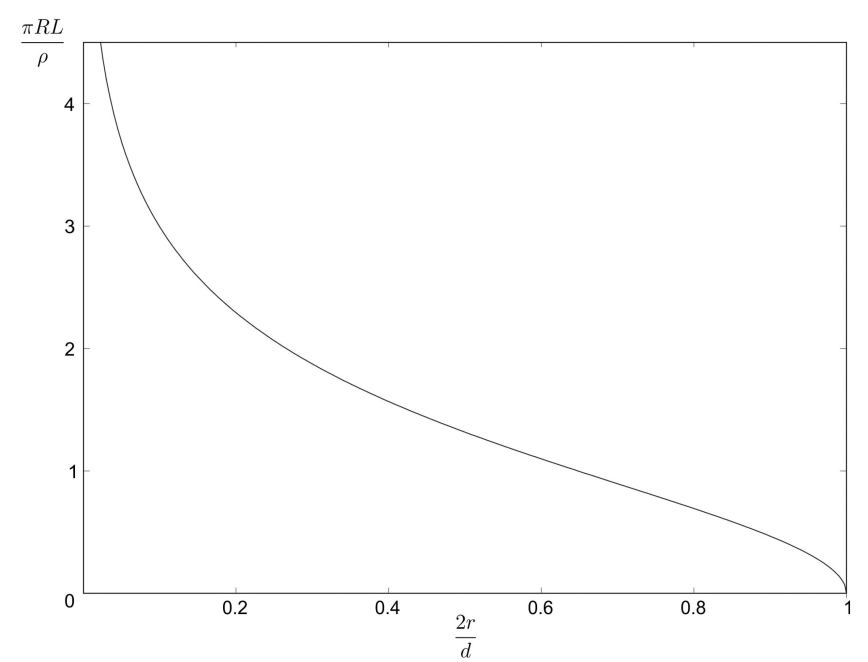

Figure 4: $\pi R L / \rho$ as function of $2 r / d$ according to Equation (9).

equal to $d(d>2 r)$. Taking into account the expression for electrical capacitance of such a system [3], we immediately get:

$$
R=\frac{\rho}{\pi l} \operatorname{arcosh}\left(\frac{d}{2 r}\right) .
$$

Thus, according to Equation (9) resistance of such a system increases nonlinearly with increasing of relative distance between wires $d / 2 r$ (see Fig. 4). For $d=2 r$ we have: $R=0$. If $d \rightarrow \infty$, then $R \rightarrow \infty$.

We can also consider the case of two balls each of radius $r$, which are at large distance $d(d \gg r)$ from each other. The electrical capacitance of each ball is $C_{0}=4 \pi \epsilon \epsilon_{0} r$. Since such a system can be treated as two capacitors connected in series, then $C=C_{0} / 2$ and

$$
R=\frac{\rho}{2 \pi r}
$$

In accordance with Equation 10 resistance $R$ does not depend on the distance between the balls. This explains the result empirically found by telegraph operators, who discovered (to their surprise) that the earth resistance between telegraph stations does not depend on the distance between them.

Using Equation 8 we may calculate the contact resistance between two conductors. Such a resistor can be considered as a single hole of radius $r$ cut into an infinitely large planar baffle of insulating material. The self-capacitance of a conducting disk is defined by $C_{0}=$ $8 \epsilon \epsilon_{0} r$. Actually, only half of $C_{0}$ is relevant $\left(C=C_{0} / 2\right)$ because for our problem the sides of the conducting disk have opposite charge signs. The total contact resistance appears as the series connection of two identical resistors ( $R_{\text {in }}$ and $\left.R_{\text {out }}\right)$, at that $R_{\text {in }}=R_{\text {out }}=\rho \epsilon \epsilon_{0} / C$. Then

$$
R=\frac{\rho}{2 r}
$$

Equation (11) was first derived by J. C. Maxwell [4. According to this equation the contact resistance increases sharply with decreasing the contact spot size.

It is interesting that Equations (3), (4) can be derived by using Equation (8). Equations (3), (4), (8) is often applied to estimate leakage resistance [5] of spherical, cylindrical and other types of capacitors. It should be noted that in many cases the problem of finding mutual capacitance is no less complicated than the problem of finding resistance. For these cases one should use the direct method for calculating resistance based on the solution of the Laplace's equation.

\section{The Trapezoid Plate Problem}

Let us consider a conducting plate of fixed length $l$ and thickness $t$. We assume that this plate has the shape of an isosceles trapezoid with curved sides and voltage $U$ is applied between its bases. We denote the lengths of the trapezoid bases as $a$ and $b$ (we put $b>a$ ) and choose two-dimensional Cartesian coordinate system as it is shown in Fig. 5 . The equation of the curved side can be approximated by a power function:

$$
y=\left(\frac{2 x-a}{b-a}\right)^{n} l,
$$

where $n$ is the exponent (power).

Using Equation (12) and the considered above method of "stack of the infinitesimal slabs" one can find the total resistance. For example:

$$
\begin{gathered}
R_{0}=\frac{\rho l}{t \sqrt{a(b-a)}} \arctan \left(\sqrt{\frac{b-a}{a}}\right) \quad(n=1 / 2), \\
R_{0}=\frac{\rho l}{t(b-a)} \ln \left(\frac{b}{a}\right) \quad(n=1), \\
R_{0}=\frac{2 \rho l}{t(b-a)^{2}}\left(b-a-a \ln \left(\frac{b}{a}\right)\right) \quad(n=2) .
\end{gathered}
$$

It should be noted that in the all cases $R_{0} \rightarrow \infty$ as $a \rightarrow 0$ (the same situation occurs if we put $r_{1} \rightarrow 0$ in

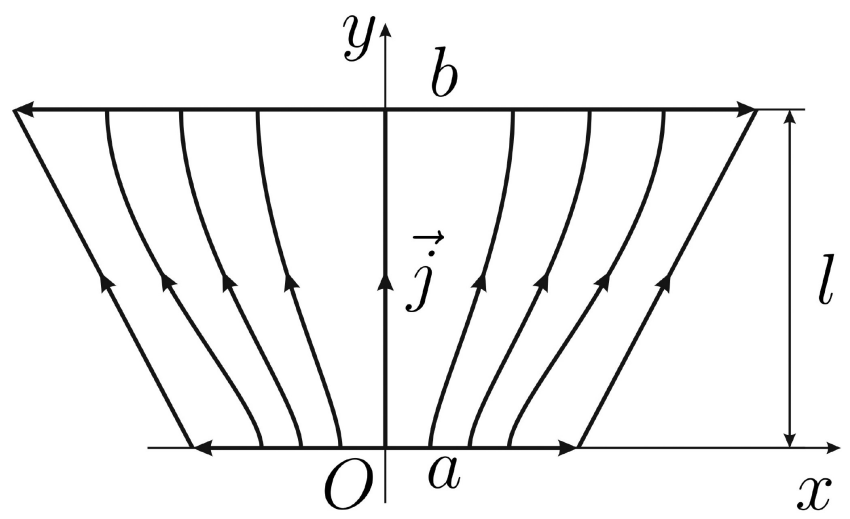

Figure 5: Geometry of the trapezoid plate problem. 


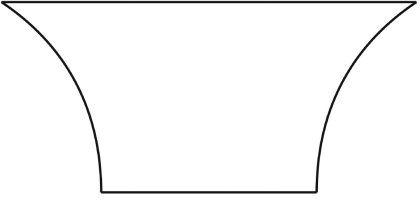

(a)

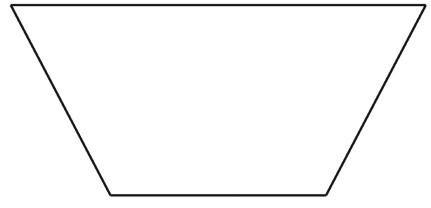

(b)

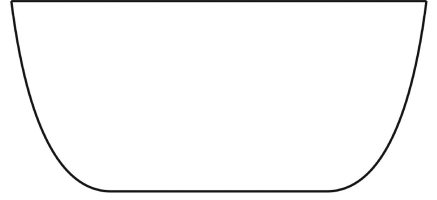

(c)

Figure 6: Three investigated geometry of the trapezoid plate. (a) $-n=1 / 2$; (b) $-n=1$; (c) $-n=2$.
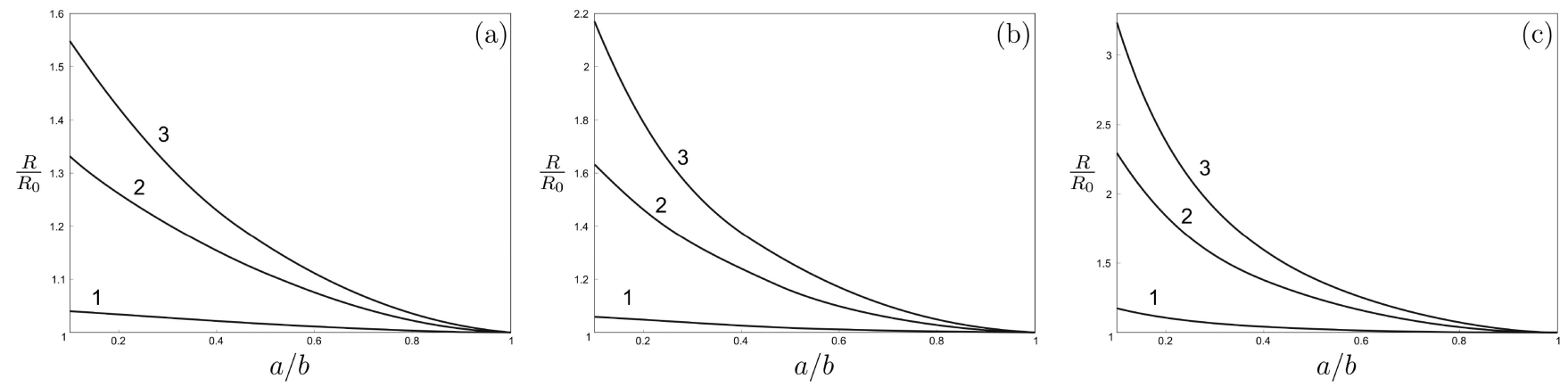

Figure 7: Dependence $R(a / b) .(1)-b / l=1 ;(2)-b / l=5 ;(3)-b / l=10$. (a) $-n=1 / 2$; (b) $-n=1 ;(\mathrm{c})-n=2$.

Equations (3), (4)). When $a \rightarrow b$ we obtain "standard" Equation (2).

The Equations (13)-(15) are derived assuming the electric field inside the conductor is homogeneous. In reality, the situation with determining the resistance for such objects turns out to be much more complicated due to the fact that the flow (electric field) lines are not straight parallel lines but are bent (see Fig. 5), so that the normal component of the electric field strength vanishes on the plate sides. Accordingly, the equipotential surfaces are also not planes perpendicular to the axis of symmetry of the trapezoid. This issue is discussed in detail in the article of Romano and Price 6. Here we continue their analysis by means of comprehensive numerical calculations performed for three cases: $n=1 / 2,1,2$ (see Fig. 6).

We start from Equation (1) and write: $U=\Phi_{1}-\Phi_{2}$, where $\Phi_{1,2}$ are the electric potentials of the truncated cone ends;

$$
I=\iint_{S} \vec{j} \cdot \mathrm{d} \vec{A}=-\frac{1}{\rho} \iint_{S} \nabla \Phi \cdot \mathrm{d} \vec{A}
$$

where the integral is taken over an arbitrary crosssection of the conductor (here we used Equation (6) and relation $\vec{E}=-\nabla \Phi)$. Thus, the solution to this problem is reduced to finding potential $\Phi$ everywhere inside the resistor. This can be done by solving the Laplace's equation $\Delta \Phi=0$ with the appropriate boundary conditions. Taking into account symmetry of our problem, we have that the electric potential depends only on coordinates $x$ and $y$ and the Laplace's equation takes form:

$$
\Delta \Phi=\frac{\partial^{2} \Phi}{\partial x^{2}}+\frac{\partial^{2} \Phi}{\partial y^{2}}=0
$$

There are the following Dirichlet boundary conditions on the bases:

$$
\begin{aligned}
& \Phi(x, 0)=\Phi_{1} \quad(-a / 2 \leqslant x \leqslant a / 2), \\
& \Phi(x, l)=\Phi_{2} \quad(-b / 2 \leqslant x \leqslant b / 2) .
\end{aligned}
$$

There is also the Neumann boundary condition at the sides of the resistor, which means that the normal to these surfaces component of the electric field strength vanishes. This condition has the form:

$$
\left.\frac{\partial \Phi}{\partial \vec{n}}\right|_{y=f(x)}=0,
$$

where $\vec{n}$ is the normal vector to the side surface; function $f(x)$ is defined by Equation 12 .

We can solve system of Equations (17)-20 numerically, applying an open-source PDE solver "FreeFem++" [7] that uses the finite element method. In Fig. 7 we plot dependences $R(a / b)$ calculated with the help of Equations (1), 160)-(20) at different values of $b / l$ and $n$. It is seen that in all cases the real (true) value of the resistance is larger than its value obtained in supposition of the homogeneous electric field. This fact can be explained on the basis of the minimum electric power principle 8 . According to this principle the currents distribute themselves inside the piece of conductor so that the electric power is as little as possible. Since 
$P=U^{2} / R$, then at fixed voltage the currents should distribute themselves to provide the maximum resistance. It means that $R>R_{0}$ always.

At fixed value of $b / l$ and $n$ the difference between $R$ and $R_{0}$ values of the resistance increases with decreasing ratio $a / r_{b}$. This difference also increases as $b / l$ and $n$ increases.

We hope that consideration of the problems described in this article will help students better grasp such an important concept as the electrical resistance.

\section{References}

[1] D. Halliday R. Resnick and J. Walker, Fundamentals of Physics (Wiley, New York, 2011), 9th ed.

[2] A. Zangwill, Modern Electrodynamics (Cambridge University Press, Cambridge, 2012), p. 278.

[3] J.D. Jackson, Classical Electrodynamics (Wiley, New York, 1975), p. 80.

[4] J.C. Maxwell, A Treatise on Electricity and Magnetism (Dover, New York, 1952).

[5] R. France, IRE Transactions on Component Parts 7, 106 (1960).

[6] J.D. Romano and R.H. Price, Am. J. Phys. 64, 1150 (1996).

[7] https://freefem.org/

[8] N.R. Sree Harsha, Eur. J. Phys. 40, 033001 (2019). 